\title{
Osteosarcomas de la región de cabeza y cuello*
}

\author{
Dra. ROCÍO LAS HERAS F. ${ }^{1}$, Int. FRANCISCA FERNÁNDEZ G. ${ }^{2}$, Drs. FELIPE CAPDEVILLE F. ${ }^{3}$, \\ MARCELO VELOSO O. ${ }^{4}$, HANS HARBST S. ${ }^{5}$, BETTINA MÜLLER ${ }^{6}$, ARTURO MADRID M. ${ }^{3}$ \\ 1 Residente de Cirugía, Clínica Alemana - Universidad del Desarrollo. \\ 2 Interno de Medicina, Clínica Alemana - Universidad del Desarrollo. \\ 3 Departamento de Cirugía de Cabeza y Cuello, Instituto Nacional del Cáncer, Clínica Alemana de Santiago. \\ 4 Departamento de Cirugía de Cabeza y Cuello, Instituto Nacional del Cáncer. \\ 5 Departamento de Radioterapia, Instituto Nacional del Cáncer, Clínica Alemana de Santiago. \\ 6. Departamento de Oncología, Instituto Nacional del Cáncer. \\ Santiago, Chile.
}

\begin{abstract}
Osteosarcoma of the head and neck area. Analysis of 12 patients

Background: Less than 10\% of osteosarcomas are located the head and neck region, mainly affecting the mandible and maxillary region. Aim: To analyze the therapeutic modality, types of reconstruction, surgical complications and survival of patients treated for osteosarcoma of the head and neck. Material and Methods: Review of medical records of 12 patients aged 17 to 34 years (6 women) treated for osteosarcoma of the head and neck between september 1998 and may 2009. Results: The localization of the tumor was maxillary in eight, mandibular three and ethmoidal in one patient. According to histologic grade, seven tumors were grade 1 , four were grade 2 and one was grade 3 . Adjuvant and neoadjuvant chemotherapy were administered to all and seven patients, respectively. The surgical treatment for maxillary tumors was maxillectomy. A reconstruction with rectum abdomini free flap was done in four patients and with dermoepidermic graft and an obturator prosthesis in three. In one patient, the defect was covered with a dental prosthesis. Treatment for mandibular tumors was mandibular excision. A reconstruction with peroneal free flap was performed in two patients and with an iliac crest graft in one. The ethmoidal tumor was resected and covered with local flaps. There were four complications. Two patients had a cutaneous fistula, one patient had a free flap partial atrophy and one patient had surgical site infection. Two patients who had positive margins died from local recurrence. Of the 10 patients with negative margins, seven are alive without recurrence, one died due to the disease, one has disseminated disease and one died from another cause. Conclusions: Our results in the treatment of head and neck osteosarcomas are consistent with published data.
\end{abstract}

Key words: Osteosarcoma head neck, tumor head neck, chemotherapy.

*Recibido el 31 de agosto de 2010 y aceptado para publicación el 7 de febrero de 2011.

No se recibió ningún apoyo financiero externo por la realización del presente trabajo.

Presentado en la 7th International Conference on Head and Neck Cancer, American Head Neck Society, San Francisco, California, julio de 2008.

Correspondencia: Dra. Rocío Las Heras F.

Vitacura 5410, Santiago, Chile.

mrociolh@gmail.com 


\section{Resumen}

Objetivos: Analizar la modalidad terapéutica, tipos de reconstrucción, complicaciones quirúrgicas y sobrevida de un grupo de pacientes tratados por esta patología. Métodos: Revisión de fichas clínicas de 12 pacientes portadores de osteosarcoma de cabeza y cuello entre septiembre de 1998 y mayo de 2009. Resultados: Edad promedio 30 años (17-34), 6 mujeres y 6 hombres. Localización fue 8 maxilar, 3 mandibular y 1 etmoidales. 7 tumores G1, 4 G2 y 1 G3. Siete pacientes recibieron quimioterapia neoadyuvante y todos adyuvante. El tratamiento de tumores maxilares fueron maxilectomías reconstruidas 4 con colgajo libre de recto abdominal, 3 con injerto dermoepidérmico más prótesis obturadora y una con prótesis dentaria. Se realizó mandibulectomía a los tumores mandibulares, 2 reconstruidos con colgajo libre de peroné y uno con injerto de cresta ilíaca. El tratamiento del tumor etmoidal fue resección craneofacial y se reparó con colgajos locales. Hubo 4 complicaciones; 2 fístulas cutáneas, una atrofia parcial de colgajo libre y una infección de herida operatoria. Dos pacientes tuvieron bordes comprometidos, quienes murieron por recidiva local. De los 10 pacientes con bordes libres, 7 se encuentran sin evidencia de recidiva, uno con enfermedad diseminada, uno fallecido por la enfermedad y uno fallecido por otra causa. Conclusiones: Nuestros resultados son consistentes con la literatura.

Palabras clave: Osteosarcoma cabeza y cuello, tumores cabeza y cuello, neoplasias cabeza y cuello.

\section{Introducción}

El osteosarcoma constituye el tumor maligno más frecuente del hueso, correspondiendo al 40$60 \%$ de todos los tumores óseos. Afecta en menos del 10\% de los casos a la región de cabeza y cuello, más comúnmente a la mandíbula y a la maxila ${ }^{1}$. El osteosarcoma de cabeza y cuello (OCC) se comporta en forma diferente de aquel que se origina en las extremidades en cuanto a que tiende a metastizar menos a los pulmones y otros sitios, pero tiene una alta letalidad asociada a enfermedad local que es difícil de controlar ${ }^{2}$. La cirugía resectiva radical ha sido el tratamiento de elección para cualquier intento curativo de la enfermedad ${ }^{3}$, ya que hasta ahora ningún tratamiento adyuvante ha sido validado por medio de estudios clínicos randomizados. En un estudio observacional retrospectivo, Guadagnolo y cols, muestran una mejoría en la sobrevida global, sobrevida libre de enfermedad y control local al usar radioterapia adyuvante en pacientes con márgenes positivos o dudosos, trabajo que constituye la base para integrar esta terapia en el manejo de los enfermos con OCC en muchos centros ${ }^{14}$.

El objetivo del presente reporte es presentar la primera experiencia nacional con respecto a esta patología y corresponde a los casos de OCC tratados en el Instituto Nacional del Cáncer entre septiembre de 1998 y mayo de 2009.

\section{Pacientes y Método}

Se realizó un análisis retrospectivo de 12 fichas de pacientes portadores de osteosarcoma en territorio de cabeza y cuello, tratados en Clínica Alemana de Santiago e Instituto Nacional del Cáncer entre septiembre de 1998 y mayo de 2009.
Se encontraron 6 mujeres y 6 hombres, promedio de edad 30 años (extremos 17 y 34). Ninguno había recibido radioterapia previa ni tenían factores mórbidos de importancia (Tabla 1).

Todos los pacientes fueron tratados según protocolo establecido por un comité multidisciplinario compuesto por cirujanos especialistas en cabeza y cuello, oncólogo médico y radioterapeuta. Todos los pacientes fueron operados por los mismos cirujanos en conjunto.

El protocolo consistió en realizar cirugía resectiva seguida de quimioterapia en caso de que fuera posible resecar el tumor dejando márgenes negativos al momento del diagnóstico. Si las condiciones quirúrgicas fueran desfavorables, ya sea por dificultad del paciente de someterse a cirugía en una fecha dada o por falta de disponibilidad de recursos hospitalarios necesarios, se indicó quimioterapia neoadyuvante y luego cirugía resectiva seguida de quimioterapia adyuvante.

Se utilizó quimioterapia adyuvante homologado al manejo estándar de osteosarcomas de extremidades ya que no existe evidencia con respecto a su utilización en osteosarcomas de cabeza y cuello, sin embargo, nos parece adecuado ofrecerle al paciente un beneficio teórico que está demostrado en otra localización.

\section{Resultados}

Se localizaron 8 tumores en la maxila, 3 en la mandíbula y 1 en etmoides. El grado histológico correspondió a 7 G1, 4 G2 y 1 G3. Todos los pacientes fueron sometidos a cirugía resectiva, 5 pacientes recibieron cirugía primaria seguida de quimioterapia y 7 pacientes cirugía posterior a tratamiento neoadyu- 
Tabla 1. Tipos de resección y reconstrucción

\begin{tabular}{|lll|}
\hline Localización & Cirugía resectiva & Reconstrucción \\
Maxila & 4 Maxilectomías & 4 Colgajos libres de recto anterior \\
& 3 Maxilectomía & IDE a lecho cruento, más prótesis obturadora \\
& 1 Maxilectomía & Prótesis dentaria \\
\hline Mandíbula & 2 Mandibulectomías parciales & 2 Colgajos libres de peroné \\
& 1 Mandibulectomía parcial & Injerto libre de cresta ilíaca \\
\hline Etmoides & 1 Resección craneofacial & Colgajos locales de rotación + prótesis \\
\hline
\end{tabular}

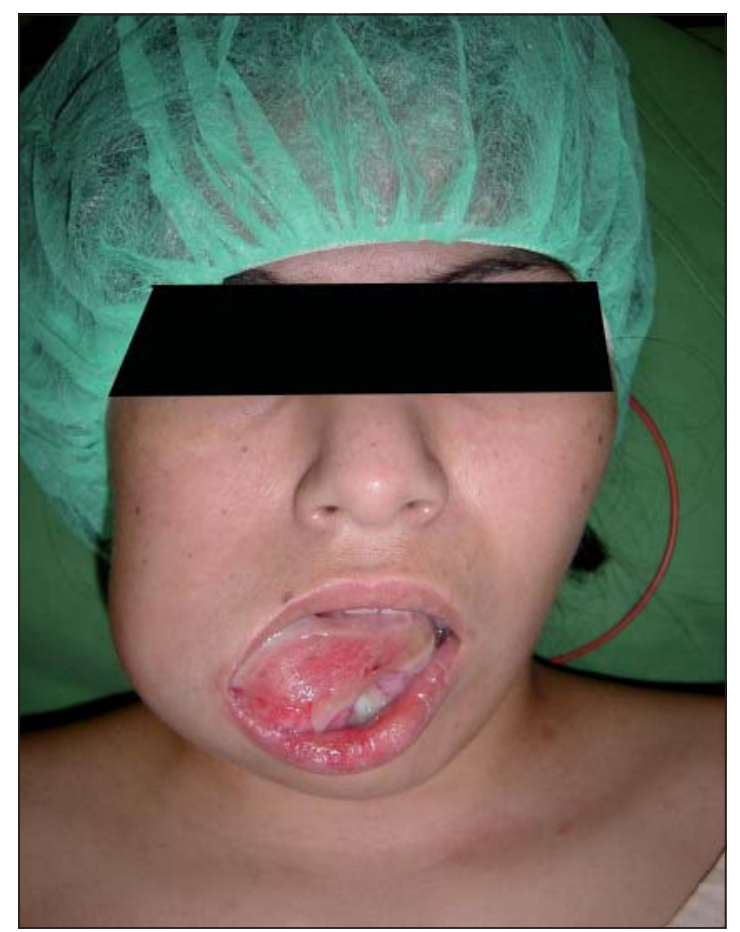

Figura 1. Paciente con osteosarcoma de mandíbula, preoperatorio.

vante. Dentro de este último grupo, un paciente fue derivado recidivado de otro centro habiendo sido tratado con quimioterapia y cirugía; otro paciente se presentó en estado avanzado al diagnóstico y se realizó cirugía de rescate posterior a quimioradioterapia neoadyuvante.

Las reconstrucciones fueron hechas en el mismo tiempo quirúrgico en 8 casos y correspondieron a 6 colgajos microvascularizados, un injerto libre de cresta ilíaca y un caso de cobertura con colgajos locales de rotación. Los colgajos libres correspondieron a 4 de recto anterior y 2 de peroné (Figuras 1, 2 y 3). El resto de las resecciones se cubrieron con injerto dermoepidérmico en 3 casos y una prótesis dentaria para una maxilectomía parcial (Tabla 1).

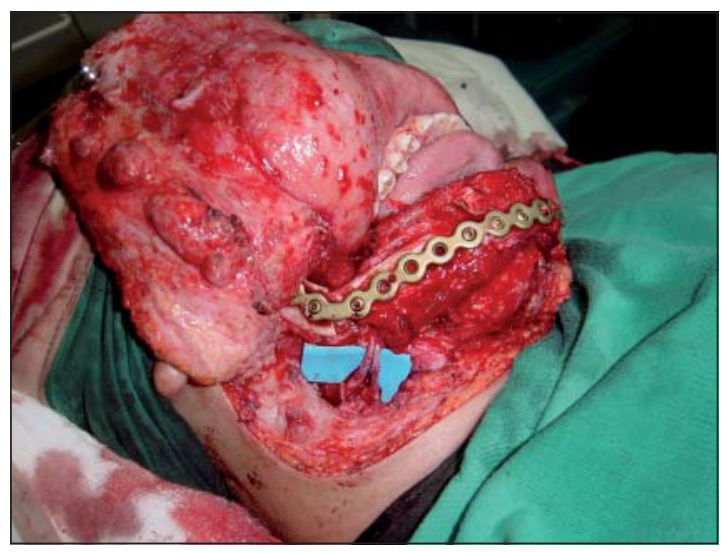

Figura 2. Colgajo libre de peroné in situ, con anastomosis a la arteria tiroidea superior a la vista.

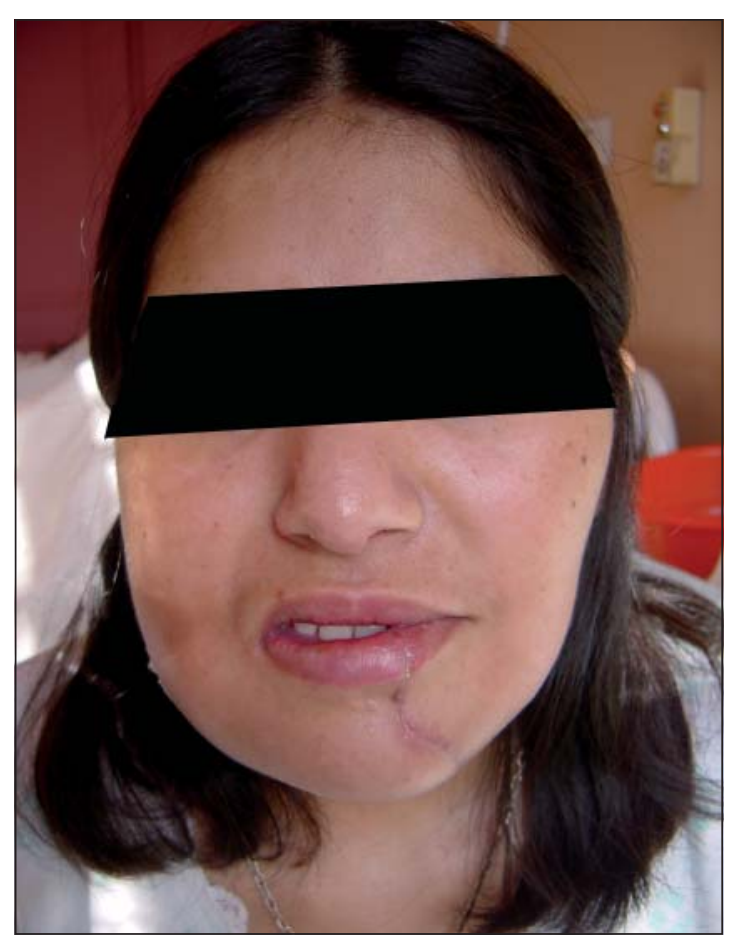

Figura 3. Resultado postoperatorio. 
OSTEOSARCOMAS DE LA REGIÓN DE CABEZA Y CUELLO

Tabla 2. Resultados del tratamiento

\begin{tabular}{|clcclc|}
\hline Pcte & Localización & Grado & Bordes & Tratamiento adyuvante & Estado \\
\hline 1 & Etmoides & 1 & - & QT - QX - QT & SER \\
\hline 2 & Maxila & 1 & + & QX - QT & MRL \\
\hline 3 & Maxila & 2 & + & QTRT - QX rescate - QT & MRL \\
4 & Maxila & 2 & - & QX - QT & SER \\
\hline 5 & Maxila & 3 & - & QT - QX - QT & MRL \\
\hline 6 & Maxila & 1 & - & QT - QX - QT & VED \\
\hline 7 & Maxila & 1 & - & QT - QX - QT & SER \\
\hline 8 & Maxila & 2 & - & QT - QX - QT & SER \\
\hline 9 & Maxila & 1 & - & QX - QT & SER \\
10 & Mandíbula & 2 & - & QTRT-QX-derivación-QX & MOC \\
\hline 11 & Mandíbula & 1 & - & QX - QT & SER \\
\hline 12 & Mandíbula & 1 & - & QX - QT & SER \\
\hline
\end{tabular}

QT: quimioterapia, QX: cirugía, RT: radioterapia; SER: sin evidencia de recidiva, MRL: muerto por recidiva local, VED: vivo con enfermedad diseminada, MOC: muerto por otra causa.

Se tuvo 4 complicaciones menores que correspondieron a 2 fístulas cutáneas con exposición de placa que se manejaron con colgajos locales de avance, una atrofia parcial de un colgajo libre de recto anterior y una infección de herida operatoria que cedió con tratamiento antibiótico.

Se tiene seguimiento completo del grupo promedio 37 meses ( 9 a 130) con una mortalidad de 4 pacientes, 3 de ellos por recidiva local y uno por causa distinta a la enfermedad. De los 3 pacientes recidivados, dos tenían bordes positivos. Se controlan 7 pacientes vivos libres de enfermedad y una paciente viva con metástasis pulmonares (Tabla 2).

\section{Discusión}

Probablemente el mayor inconveniente en el estudio del OCC es el muy escaso número de pacientes a los que afecta, lo que hace prácticamente imposible confeccionar estudios prospectivos. Por lo anterior, el conocimiento generado ha sido en base a estudios retrospectivos con números de pacientes variables que involucran muchos años, durante los cuales los esquemas de tratamiento han variado en forma acorde. Actualmente, muchos centros basan su manejo en las pautas establecidas de osteosarcomas de extremidades, con las variaciones particulares para cada caso, que son resueltas en comités de especialistas, tal como se realiza en nuestro centro. También se utiliza frecuentemente la experiencia publicada de otros centros, pero cabe hacer notar que no son más que reportes. La publicación más grande incluye 496 casos, pero es una recolección de pacientes de diversos centros donde las terapias son diferentes ${ }^{4}$, por lo que su mayor aporte es probablemente la información epidemiológica acerca de la población afectada.

Nuestros pacientes son comparables a series internacionales ${ }^{5,6}$ en cuanto a sus características demográficas. Los OCC afectan principalmente a sujetos entre la tercera y la cuarta década de la vida, en contraste con los osteosarcomas de las extremidades que afectan a adolescentes. Los hombres son levemente más afectados que las mujeres en nuestra serie. La mandíbula y la maxila fueron en nuestro estudio los sitios más frecuentes de presentación de la enfermedad. Se han descrito factores de riesgo en otras publicaciones como la radiación previa ${ }^{7}$, retinoblastoma ${ }^{8}$, síndrome de Li-Fraumenii ${ }^{9}$ y síndrome de Rothmund-Thomson ${ }^{10}$, lo que no vimos reflejado en nuestro estudio.

Nos parece de vital importancia contar con un equipo entrenado en reconstrucción de lesiones complejas de cabeza y cuello para ser capaces de resecar el tejido que sea necesario y no limitar la resección a las capacidades reconstructivas. Esta práctica puede dejar pacientes con márgenes positivos de manera innecesaria, lo que, como sabemos, entrega los peores resultados oncológicos. En nuestra serie sólo dos pacientes tuvieron bordes positivos y ambos fallecieron por recidiva local, mientras que de los diez restantes, sólo uno falleció por esta causa (Tabla 2).

Nuestros resultados de tratamiento también son comparables a lo descrito en la literatura. Pudimos observar una sobrevida de $69,2 \%$ en el grupo completo a 3 años y el factor más frecuente que se 
relacionó de manera favorable con la sobrevida fueron los márgenes quirúrgicos negativos como se ha reportado en diversos centros ${ }^{11-13}$. Además pudimos ver en nuestra serie que el grado tumoral no influyó en la sobrevida, acorde con lo descrito por algunos grupos, aunque la gran mayoría de los estudios señalan lo contrario ${ }^{14,15}$, donde el mayor grado tumoral disminuye la sobrevida.

Tradicionalmente la quimioterapia se ha considerado beneficiosa, basándose principalmente en el estudio de Smeele y cols ${ }^{13}$, en 1997 que mostró mejoría en la sobrevida libre de enfermedad y en la sobrevida global independiente de los márgenes. Sin embargo, publicaciones más recientes han concluido lo contrario ${ }^{14}$. Debe considerarse, sin embargo, que la heterogeneidad de los grupos de pacientes y la falta de uniformidad de terapias hacen difícil la interpretación de los datos.

Recientemente, un equipo colaborativo del MD Anderson Cancer Center, ha publicado el estudio tal vez más importante con respecto a la utilidad de la radioterapia en el manejo del $\mathrm{OCC}^{14}$, que a pesar de ser retrospectivo, incluye un gran número de pacientes que permite aventurar importantes conclusiones. En este trabajo se incluyeron 119 pacientes que recibieron cirugía radical con intención curativa; de ellos, el 77\% fueron sometidos solamente a cirugía, mientras 23\% recibieron radioterapia mediana de 60 Gy independiente de los bordes positivos o negativos tras la resección. Pudieron ver que la radioterapia añadida a la cirugía mejora la sobrevida total (80 vs 31\%), la sobrevida libre de enfermedad (80 vs $35 \%$ ) y disminuye la recurrencia local (75 vs $24 \%$ ) en pacientes con márgenes dudosos o positivos. La radioterapia no presentó diferencias significativas en pacientes con márgenes negativos tras la cirugía. Los resultados de este estudio han hecho a varios centros añadir la radioterapia al manejo quirúrgico estándar de estos pacientes.

\section{Referencias}

1. Wanebo HJ, Koness RJ, MacFarlane JK, Eilber FR, Byers RM, Elias EG, et al. Head and Neck Sarcoma: report of the Head and Neck Sarcoma Registry. Head Neck 1992;14:1-7.

2. Dahlin DC, Unni KK. Osteosarcoma Bone Tumors. Springfield Ill: Charles C. Thomas Publisher, 1986:269307.
3. Sturgis EM, Potter BO. Sarcomas of the head and neck region. Curr Opin Oncol. 2003;15:239-52.

4. Smith RB, Apostolakis LW, Karnell LH, Koch BB, Robinson RA, Zhen W, et al. National Cancer Data Base Report on Osteosarcoma of the Head and Neck. Cancer 2003;98:1670-80.

5. Mark RJ, Sercarz JA, Tran L, Dodd LG, Selch M, Calcaterra TC. Osteosarcoma of te head and neck. The UCLA experience. Arch Otolaryngol Head Neck Surg. 1991;117:761-6.

6. Oda D, Bavisotto LM, Schmidt RA, McNutt M, Bruckner JD, Conrad EU 3rd, et al. Head and neck osteosarcoma at the University of Washington. Head Neck. 1997;19:513-23.

7. Tucker MA, D’angio GJ, Boice JD Jr, Strong LC, Li FP, Stovall M, et al. Bone sarcomas linked to radiotheray and quemotherapy in children. $\mathrm{N}$ Eng $\mathrm{J}$ Med. 1987;317:588-93.

8. Hansen MF, Koufos A, Gallie BL, Phillips RA, Fodstad O, Brøgger A, et al. Osteosarcoma and retinoblastoma: a shared chromosomal mechanism revealing recessive predisposition. Proc Natl Acad Sci USA. 1985;82:621620.

9. Li FP, Fraumeni JF Jr, Mulvihill JJ, Blattner WA, Dreyfus MG, Tucker MA, et al. A cancer family syndrome in twenty-four kindreds. Cancer Res. 1988;48:5358-62.

10. Leonard A, Craft AW, Moss C, Malcolm AJ. Osteogenic sarcoma in the Rothmund - Thomson syndrome. Med Pediatr Oncol. 1996;26:249-53.

11. Ha PK, Eisele DW, Frassica FJ, Zahurak ML, McCarthy EF. Osteosarcomas of the head and neck: a review of the Johns Hopkins experience. Laryngoscope 1999;109:964-9.

12. Kassir RR, Rassekh CH, Kinsella JB, Segas J, Carrau RL, Hokanson JA. Osteosarcoma of the head and neck: metanalisis of non randomized studies. Laryngoscope 1997;107:56-61.

13. Smeele LE, Kostene PJ, van der Waal I, Snow GB. Effect of chemotherapy on survival of craneofacial osteosarcoma: a systematic review of 201 patients. J Clin Oncol. 1997;15:363-7.

14. Guadagnolo BA, Zagars GK, Raymond AK, Benjamin RS, Sturgis EM. Osteosarcoma of the jaw/craneofacial region. Outomes after multimodality treatment. Cancer 2009;115:3262-70.

15. Laskar S, Basu A, Muckaden MA, D’Cruz A, Pai S, Jambhekar N, et al. Osteosarcoma of the head and neck region: lessons learned from a single institution experience of 50 patients. Head Neck 2008;30:1020-6. 